\title{
A Physio-anthropological Approach in Evaluation of Human Adaptability to Living Environment: In the Case of Artificial Light Environment
}

\author{
Akira Yasukouchi \\ Department of Physiological Anthropology, Kyushu University
}

\begin{abstract}
Attention has been focused on human adaptability to human-adopted artificially modern and comfortable environments which may not always match the adaptability by the physiological state of humans. This discrepancy was found to induce a slight tension in the human body on evaluation with a physio-anthropological approach. Although a standard methodological approach has yet to be established, the present study attempted to evaluate human adaptability to artificial light environment, based on evaluations from three major possible perspectives: a) central nervous system, b) autonomic nervous system and c) biological rhythm. In order to detect the slight tension, human volunteers were exposed to an artificial light environment, and the appropriate physiological parameters were then examined from photic signal pathways in a brain. The findings revealed that a higher color temperature of fluorescent lamps induced a slight tension, although many other factors remained to be elucidated. $J$ Physiol Anthropol Appl Human Sci 24 (4): 307-312, 2005 http://www.jstage.jst.go.jp/browse/jpa
\end{abstract}

[DOI: 10.2114/jpa.24.307]

Keywords: physio-anthropological approach, human adaptability, slight tension, light, fluorescent lamp, biological rhythm

\section{Concept of the Physio-anthropological Approach}

Physiological anthropology is a field that deals with humans mainly living in highly technological environments. Many different industrial products that furnish convenience and comfort in our lifestyle mold the living environment we dwell in. However, when the evolutionary process of the humans is taken into consideration, many important problems remain unresolved. As human beings spent more than $99.8 \%$ of the 5 million-old evolutionary history on gather-and-hunt, the human biological system had therefore adapted to a natural living environment molded more by natural forces. However, the present artificial environment, which has been modified and molded by sophisticated technological advents in a moment on a scale of the evolutionary history, presents many challenges to the intrinsic physical resources of human beings.

As the human brain has a greater pleasant center and neocortex among primates (Eccles, 1989), humans adapt and accommodate more intelligently to their physical needs with versatility. However, human body occasionally unable to biologically cope with such living environments. Along this line of development, we focus on the balance between braincontrolled voluntary intentions/behaviors and involuntary physiological responses of the body to external stimuli. By rendering the human body to appropriately respond to the living environment, this balance ensures not only human survival but furnishes true health and comfort in the living environment as well.

When the human body does not appropriately adapt to the environment in the presence of stress, the system responds by manifesting certain physiological changes which can be detected and monitored, even if such stress is slight and invisible. As such, if any tension can be detected in a given environment, means and solutions to improve and neutralize the environmental impact to mold a more conducive environment for human accommodation may be formulated.

Why do we have to pay attention to stress, a trivial and apparently non-harmful factor? There are various facets of stress that we have to avoid, adapt and accommodate and be able to live with in our daily life; e.g., the issues of economy, education, livelihood, work, pleasant and unpleasant mental inputs, etc. Therefore, we need a kind of accommodative capacity to cope with such stresses/issues. However, when subtle technologically modified environment-induced tensions continue to exert and accumulate in our body system, the accommodative capacity is reduced over time, and said tension has to be released in the form of irritation and, in certain circumstances, mental panic. If this mentally unstable and stress-loaded condition is unattended to and allowed to prevail, not only physical and/or mental fatigue but also disorders in the biological rhythm (BR) affecting growth, learning ability, attention, hormonal and other endogenous secretions, anxiety, 
etc. will develop over time.

\section{Methodological Approach}

Although a standard methodology to evaluate and detect such environmentally induced tension has yet to be established, certain possible approaches were adopted in this study. The amygdala, which receive all sensory signals including nonperceptive ones from the environment to cope with the integrated information for biological evaluation, and process these inputs to subsequently project signals to the hypothalamus, where output signals to physiological effectors eventually control to mold an adaptive behavior (DeLoux, 1996). Therefore, detectable changes in physiological but not psychological parameters could be monitored when the slight tension did exist.

Of the living system, approaches with the application of 3 major perspectives may theoretically be feasible to evaluate said tension; viz., the (a) central nervous system (CNS), (b) autonomic nervous system (ANS) and (c) biological rhythm (BR). Besides approaches (a), (b) and (c), further investigations of the sympathetic-adrenomedullary and pituitary-adrenocortical responses should also be endeavored as useful tools to detect said tension.

\section{Approach (a): CNS}

It has been known that the theoretical relationship between the tonic arousal level and the efficiency of performance portrays an inverted U-shape curve (Hebb, 1949; Olst and Orlebeke, 1967). The tonic arousal level might be replaced into cortical arousal level as shown in Fig. 1. Performance increases with elevations in the cortical arousal level; however, when the arousal level increases beyond the optimum (threshold level), performance decreases. The latter manifests a phase where the arousal level has attained an excited condition, such as the state of being irritated or nervous. The early phase manifested by this condition might be considered as a state where a slight tension had been molded unawared. Contingent negative variations $(\mathrm{CNV})$, employed as the index of cortical arousal levels, might serve as a possible evaluation parameter for said tension induced in CNS because performance can be represented by the response time based on CNV paradigm.

\section{Approach (b): ANS}

Although theoretical relationship (a) has been anticipated, there also might prevail another relationship between the autonomic nerve tone and its output, i.e. the heart rate. Heart rate controlled by the balance between sympathetic and parasympathetic nervous tones is always changing to yield an optimal level accommodating spontaneous variations in behaviors or responses to environmental conditions.

According to typical relationships between the cardiac related autonomic nervous tones and heart rate (Fig. 2), the heart rate at a resting condition is usually controlled by the parasympathetic tone; however, when certain strains occurred

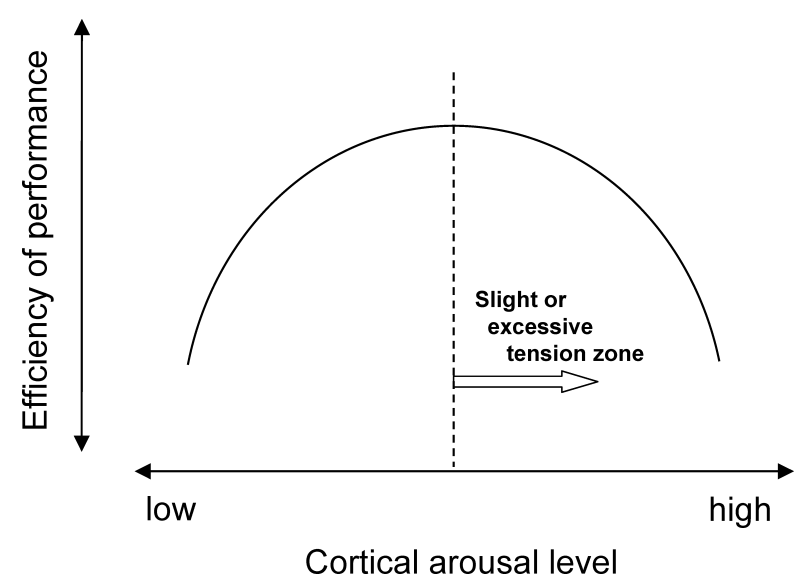

Fig. 1 Theoretical relationship between efficiency of performance and cortical arousal level.

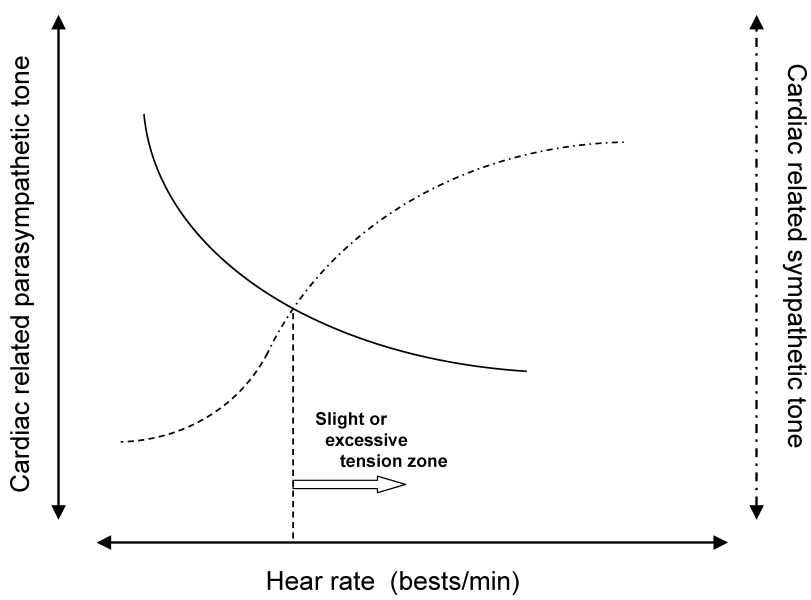

Fig. 2 Theoretical relationship between cardiac related autonomic nervous tones and heart rate.

in the body system, the sympathetic tone then predominates to control or increase the heart beat. This threshold (Fig. 2) is a critical point, above which the sympathetic tone becomes dominant to manifest a slightly excessive tension zone.

\section{Approach (c): BR}

Many biological functions, such as body temperature regulation, hormonal secretion, immune responses and other physiological parameters, synchronize a certain rhythm with an approximately 24 -hour cyclic pattern; i.e. the circadian rhythm. A rhythm is characterized by amplitude, or the difference between the peak level (or nadir) and the mesor (the midpoint of the range of levels), and peak times. As lighting is a primary environmental stimulus with which the circadian system is synchronized, changes in the circadian rhythm would serve as useful parameters for evaluating the lighting effect on tensions exerted on the body system, in view of examining the deviations of amplitude and/or timing of the peak from the normal level. However, a critical degree of the deviation should be taken into consideration for evaluations, according to the purpose of the study. 


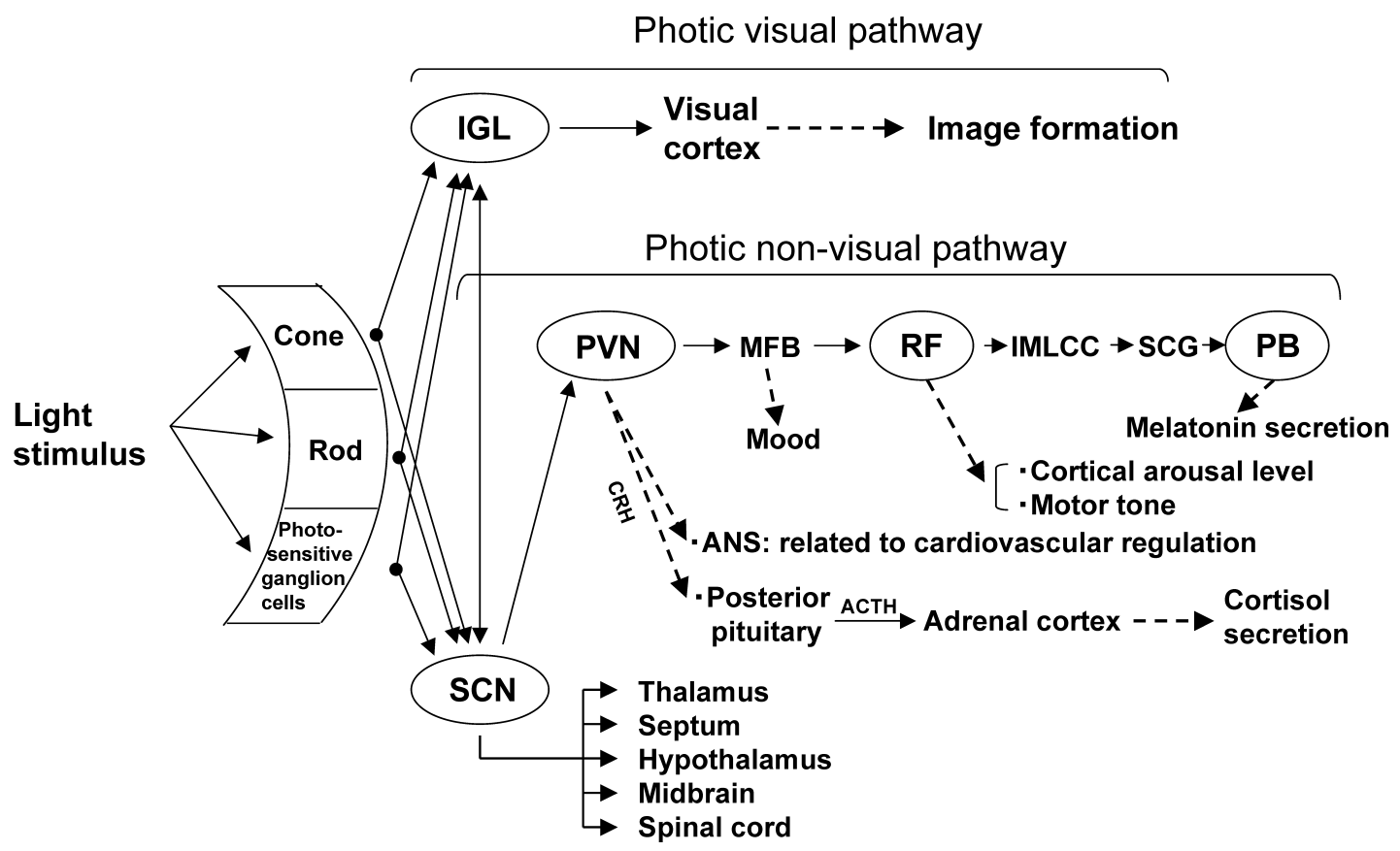

Fig. 3 Two photic pathways in the brain; visual and non-visual. IGL: Intergeniculate leaflet, SCN: Suprachiasmatic nucleus of hypothalamus, PVN: Paraventricular nucleus of hypothalamus, MFB: Medial forebrain bund, RF: Reticular formation, IMLCC: Intermediolateral cell column, SCG: Superior cervical ganglion, PB: Pineal body, CRH: Corticotropic releasing hormone, ACTH: Adrenocorticotropic hormone.

\section{In the Case of Artificial Light Environment}

\section{(1) Physiological responses to a lighted environment}

In order to appropriately evaluate human adaptability to lighting, we need to select effective physiological parameters to detect if a certain tension exists in a body. In this aspect, the photic signal currency serves to predict the optimal physiological parameters.

There are two major photic pathways (Fig. 3): viz., a visual and a non-visual pathway. Light stimulus is first perceived on the retina. Mainly the cone and rod project to visual cortex via the intergeniculate leaflet (IGL) to actualize image formation, while the cone, rod and photosensitive ganglion cells project impulses to the suprachiasmatic nucleus (SCN) of the hypothalamus; i.e., the retinohypothalamic tract (RHT) (Klein et al., 1983; Berson et al., 2002; Hatter et al., 2002). The bilateral SCN are considered to generally regulate the physiological rhythm as a biological clock. SCN relay photic signal to pineal body and regulate melatonin secretion. In coursing to the pineal body, a signal transmits through several important sites, where the light stimulus might affect physiological functions; viz., a) the paraventricular nucleus (PVN) of hypothalamus displays functions related to the ANS with reference to cardiovascular regulation and related to cortisol secretion via the posterior pituitary and adrenal cortex; b) the medial forebrain bund (MFB) affecting mood; and c) the reticular formation (RF) affecting the cortical arousal level when the photic signal/stimulus ascending the cerebrocortex which might also affect muscle tension via the spinal motor neurons when the signal reflected by cortical arousal level descending from cerebrocortex to spinal cord. SCN deliver photic visual and non-visual signals to non-visual pathways via a connection with IGL, and diffuse projections to other hypothalamic nuclei such as the septum, thalamus, midbrain and spinal cord (Klein et al., 1991), albeit non-visual effect and the functional roles have yet to be elucidated. However, it has been known that lighting affects numerous functions associated with the ANS including body temperature regulation, almost all hormonal secretions, metabolisms, immune system, reproduction and higher cognitive and emotional functions. Particular attention has to be focused on the non-visual parameters that include cortical arousal level, cardiovascular autonomic nerve tones, body temperature rhythm and melatonin secretion because we have confirmed that these physiological parameters were affected on exposure to artificial lights (Yasukouchi and Ejima, 1998; Sato and Yasukouchi, 1999; Yasukouchi et al., 2000; Yasukouchi and Ishibashi, 2005).

\section{(2) Physical factors of a lighted environment to examine human adaptability}

Lighting factors affecting human body functions should be considered in evaluations of human adaptability to a living environment where light is equipped.

Natural light is composed of wavelengths within a range of the electromagnetic spectrum (visible: $380-780 \mathrm{~nm}$ ). The spectra and intensities of natural lights change according to the time of day and seasons. Therefore, intensity and spectral composition of lighting should be emphasized as factors affecting the physiological aspects in humans. 
This interpretation is partially similar to the case of artificial lighting because changes in intensity depend on the building space, although the magnitude is considered very small on a logarithmic scale, and the spectral composition is different compared with those of natural light. Fluorescent lamps, which are called three-band type lamp with three-peak energy (i.e., red, green and blue regions), are popularly employed in Japan for lighting in buildings and even at home. The light sources of daylight, white light and warm-white light, for example, are determined by the energy combination of red, green and blue. The effects of intensity and spectral composition of artificial lamps have to be examined to evaluate human adaptability in relation with the time, duration and temporal pattern of exposure (CIE, 2004).

In Japan, the number of office workers represents more than two-thirds of the work force. As many work in buildings and underground facilities, they are seldom exposed to sunlight while at work during daytime. However, many workers on night-shifts are exposed to bright artificial lighting, inducing multifaceted physiological changes due to phase-shift of rhythm and melatonin suppression (Zeitzer et al., 2000; Wright et al., 2001). As humans are originally adapted to natural lighting during daytime and are exposed to darkness or dim lights of moon and fire at night, these intrinsic properties have to be taken into consideration when the effects of artificial lighting on the human body are examined in daytime and/or night-time, respectively.

\section{Physio-anthropological Evaluations of Human Adaptability to a Lighted Environment}

The evaluation of human adaptability to a lighted environment was attempted according to the concept of a physio-anthropological approach. Some results were summarized in a previous report (Yasukuchi and Ishibashi, 2005). Typical evaluations based on the CNS by Iwakiri et al. (1997a) measured CNV in 7 young male adults under different color temperature conditions of $3000 \mathrm{~K}, 5000 \mathrm{~K}$ and $7500 \mathrm{~K}$, and a significantly higher cortical arousal level with a significantly prolonged response time were indicated at $7500 \mathrm{~K}$ (daylight) compared with those under warm-white light $(3000 \mathrm{~K})$. Deguchi and Sato (1992) have first investigated the effects of color temperature of fluorescent lamps on $\mathrm{CNV}$, and noted a similar finding; viz., the amplitude of early component of CNV was significantly greater at $7500 \mathrm{~K}$ than at $3000 \mathrm{~K}$ without difference in response time between both conditions. Inoue and Yasukouchi (1993) have further reconfirmed the result of Deguchi and Sato (1992) by measuring auditory event-related potentials at a thermally neutral condition (but not at ambient temperature conditions of $15^{\circ} \mathrm{C}$ and $35^{\circ} \mathrm{C}$ ). Collectively, these reports revealed that daylight would enhance cortical arousal levels without improving performance. It was suggested that daylight could cause a slight tension in a thermally neutral condition in view of evaluations from the CNS.
Mukae and Sato (1992) have investigated the effects of fluorescent lamps on autonomic nervous tones by focusing on heart rate variability. The lighting conditions were designated at three levels of color temperature $(3000 \mathrm{~K}, 5000 \mathrm{~K}$ and $6700 \mathrm{~K})$ and three levels of illuminance $(100 \mathrm{~lx}, 300 \mathrm{~lx}$ and $9001 \mathrm{x})$. The results demonstrated that both cardiac related parasympathetic and sympathetic nerve tones were significantly enhanced under $6700 \mathrm{~K}$ independent of illuminance levels. Ishibashi et al. (1997) have further reported the effects of the combined environmental factors of ambient temperature, color temperature, and noise on heart rate variability. A significant interaction between color temperature and noise condition indicated that the cardiac related sympathetic tone was higher at $7500 \mathrm{~K}$ than at $5000 \mathrm{~K}$ and $3000 \mathrm{~K}$ only under background noise condition. According to Kobayashi and Sato (1992), the diastolic blood pressure under $6700 \mathrm{~K}$ lighting was significantly enhanced. Although these studies were performed in daytime, results obtained by Tsutsumi et al. (2002) during night-time demonstrated convergent color temperature effects on heart rate variability and blood pressure. The condition of $6700 \mathrm{~K}$ lighting caused significantly increased cardiac related sympathetic tone in a simulated living room and significantly greater systolic blood pressure in a simulated bedroom compared with conditions of $5000 \mathrm{~K}$ and $3000 \mathrm{~K}$. These results showed that a higher color temperature induced a slight tension in both daytime and nighttime. However, the higher color temperature effects are only observed under conditions where the ambient temperature and noise are nonstress-imposing.

In a previous study by Morita and Tokura (1996) on the effect of color temperature of fluorescent lamps exposed before sleep on body temperature and melatonin rhythm during sleep at night, subjects were instructed to look at lighting sources for at least $1 \mathrm{~min}$ at 10 -min intervals during exposure to lighting before sleep. The result clearly indicated that the amplitude of body temperature rhythm and the amount of melatonin secretion during sleep were significantly smaller under daylight than under warm-white condition. Moreover, Higashihara et al. (2002) have demonstrated findings parallel to the results of Morita and Tokura (1996) even when the subjects were instructed not to look at the lighting sources at all. Both studies converge on the fact that a higher color temperature suppressed the amplitude of body temperature rhythm and the amount of melatonin secretion during sleep at night. However, when elderly subjects are investigated in their daily life by Hashitomi et al. (personal communication), no significant effects of color temperature exposed before sleep on melatonin secretion are noted during sleep. This discrepancy may be accounted by attenuation in transmitting short wavelengths in the elderly (Brainard et al., 1997).

All in these studies based on approaches of CNS and ANS and BR have revealed that daylight tends to cause said slight tension in our human living system. However, many adaptability-related factors remain to be resolved in evaluating human adaptability to a lighted environment. Iwakiri and 
Yasukouchi (1997b) have demonstrated that the cortical arousal level was significantly elevated under $6500 \mathrm{~K}$ than $3000-3700 \mathrm{~K}$ lightings with a color rendering property of $\mathrm{Ra}$ 88 , albeit null effects were evoked when color rendering properties of $\mathrm{Ra} 72-75$ and $\mathrm{Ra} 95-99$ were employed. This implies that the cortical arousal level is influenced by a difference in spectral distribution rather than the color temperature scale per se. In a study on diurnal changes of physiological responses to the light, Arikura et al. (2004) have indicated that the results of comparisons of heart rate variability responses between blue and red lights were different, depending on when the value was measured, morning or evening.

Apart from lighting factors affecting the human body, we have observed existence of a human variation in response to different light stimuli. Hazama et al. (2004) have furnished evidence of substantial variations in pupil response to lightings with different spectral compositions, which might also have a relation to individual difference in melatonin suppression. Higuchi et al. (2005) have further indicated two of 17 subjects showing no melatonin suppressions even after exposure to bright light at night. These studies are just some of the examples that typically manifest individual characteristics, however, which becoming important issues in the field of physiological anthropology because polymorphism in physiological traits is an important keyword to evaluating human adaptability in relation with functional potentiality and whole-body coordination. Along this line, physioanthropological approaches have to be adopted for appropriate evaluations of human adaptability to lighted environments.

Acknowledgements This study was supported in part by the Grant-in-Aid for Scientific Research (S) (grant no. 15107006) from the Japanese Ministry of Education, Culture, Sports, Science, and Technology.

\section{References}

Arikura S, Horinouchi K, Kozaki T, Hazama T, Yasukouchi A (2004) Diurnal and seasonal variation of responses to different wavelength lights in cardiac autonomic nervous system. J Physiol Anthropol Appl Human Sci 23: 176

Berson DM, Dunn FA, Takao M (2002) Phototransduction by retinal ganglion cells that set the circadian clock. Science 295: 1070-1073

Brainard GC, Rollag MD, Hanifin JP (1997) Photic regulation of melatonin in humans: Ocular and neural signal transduction. J Biol Rhythms 12: 537-546

CIE Technical Report (2004) Ocular lighting effects on human physiology and behaviour. CIE: 158

Deguchi T, Sato M (1992) The effect of color temperature of lighting sources on mental activity level. Ann Physiol Anthropol 11: 37-43

Eccles JC (1989) Evolution of the brain: Creation of the self. Routledge, London and New York
Hatter S, Liao HW, Takao M, Berson DM, Yau KW (2002) Melanopsin-containing retinal ganglion cells: architecture, projections, and intrinsic photosensitivity. Science 295: 1065-1070

Hazama T, Kozaki T, Yasukouchi A (2005) Pupil responses to light with different spectral composition. J Physiol Anthropol Appl Human Sci 24: 188

Hebb D (1949) The organization of behavior. Wiley, New York Higashihara Y, Tsutsumi Y, Kozaki T, Kitamura S, Ueda S, Horinouchi K, Ishibashi K, Yasukouchi A (2002) Effects of color temperature of lighting sources on body temperature in the living room and bedroom at night. 6th Int Congress of Physiol Anthropol: 35

Higuchi S, Motohashi Y, Maeda T, Ishibashi K (2005) Relationship between individual difference in melatonin suppression by light and habitual bedtime. J Physiol Anthropol Appl Human Sci 24: 419-423

Inoue S, Yasukouchi A (1993) Effects of color temperature and ambient temperature on brain event related potentials. Ann Physiol Anthropol 12: 110

Ishibashi K, Takeyasu H, Tamura H, Watanuki S, Yasukouchi A (1997) Effects of combined environment of ambient temperature, color temperature and noise on heart rate variability. Appl Human Sci J Physiol Anthrop 16: 225

Iwakiri K, Watanuki S, Yasukouchi A, Tochihara Y (1997a) The effect and after-effect of light-source color on early component of CNV. Jpn J Physiol Anthrop 2: 31-37 [In Japanese]

Iwakiri K, Yasukouchi A (1997b) The effects of color temperature and color rendering properties of light sources on arousal level. Jpn J Physiol Anthropol 2: 173-178 [In Japanese]

Klein DC, Smoot R, Weller JL, Higa S, Markey SP, Creed GJ, Jacobowitz DM (1983) Lesions of the paraventricular nucleus area of the hypothalamus disrupt the suprachiasmatic Spinal cord circuit in the melatonin rhythm generating system. Brain Res Bull 10: 647-652

Klein DC, Moore RY, Reppert SM (1991) Suprachiasmatic nucleus: The mind's clock. Oxford University Press, Oxford

Kobayashi H, Sato M (1992) Physiological responses to illuminance and color temperature of lighting. Ann Physiol Anthropol 11: 45-49

Morita T, Tokura H (1996) Effects of lights of different color temperature on the nocturnal changes in core temperature and melatonin in Humans. Appl Human Sci J Physiol Anthropol 15: 243-246

Mukae H, Sato M (1992) The effect of color temperature of lighting sources on the autonomic nervous functions. Ann Physiol Anthropol 11: 533-538

LeDoux J (1996) Emotional networks and motor control: a fearful view. In Holstege G, Bandler R, Saper CB eds. The emotional motor system. Progress in brain research vol. 107. Elsevier

Sato M, Yasukouchi A (1999) Non-visual effects of room illumination. In Sato M, Tokura H, Watanuki S eds. Recent 
advances in physiological anthropology. Kyushu University Press, Fukuoka

Tsutsumi Y, Kitamura S, Kozaki T, Ueda S, Higashihara Y, Horinouchi K, Noguchi H, Ishibashi K, Yasukouchi A (2002) Effects of color temperature of lighting in the living room and bedroom at night on autonomic nerve activity. 6th Int Congress of Physiol Anthropol: 36

Van Olst EH, Orlebeke JF (1967) An analysis of the concept of arousal. Nederlands Tijdschrift voor de Psychologie 22: 583-603

Wright KP Jr, Hughes RJ, Kronauer RE, Dijk DJ, Czeisler CA (2001) Intrinsic near-24-h pacemaker period determines limits of circadian entrainment to a weak synchronizer in humans. Proc Natl Acad Sci USA 98: 14027-14032

Yasukouchi A, Ejima H (1998) The effects of color temperature of light sources on the arousal level and postural change with different mental tasks. Proc Second Int Conference on Human-Environment System: 247-250

Yasukouchi A, Yasukouchi Y, Ishibashi K (2000) Effects of color temperature of fluorescent lamps on body temperature regulation in a moderately cold environment. J Physiol Anthropol Appl Human Sci 19: 125-134

Yasukouchi A, Ishibashi K (2005) Non-visual effects of the color temperature of fluorescent lamps on physiological aspects in humans. J Physiol Anthropol Appl Human Sci 24: $41-43$

Zeitzer JM, Dijk DJ, Kronauer R, Brown E, Czeisler CA (2000) Sensitivity of the human circadian pacemaker to nocturnal light: melatonin phase resetting and suppression. $\mathrm{J}$ Physiol 526 (Pt 3): 695-702

Received: January 31, 2005

Accepted: March 15, 2005

Correspondence to: Akira Yasukouchi, Department of Physiological Anthropology, Kyushu University, 4-9-1 Shiobaru Minami-ku, Fukuoka 815-8540, Japan

Phone: +81-92-553-4530

Fax: +81-92-553-4530

e-mail: yasukouc@design.kyushu-u.ac.jp 